\title{
Le transfert technique et ses avatars : le cas de la Russie
}

Technical transfer and avatars : the Russia case

Irina Gouzévitch

\section{CpenEdition}

\section{Journals}

Édition électronique

URL : http://journals.openedition.org/dht/1158

DOI : $10.4000 /$ dht. 1158

ISSN : 1775-4194

Éditeur :

Centre d'histoire des techniques et de l'environnement du Cnam (CDHTE-Cnam), Société des élèves du CDHTE-Cnam

Édition imprimée

Date de publication : 1 décembre 2007

Pagination : $14-23$

ISBN : 978-2-9530779-0-2

ISSN : 0417-8726

Référence électronique

Irina Gouzévitch, « Le transfert technique et ses avatars : le cas de la Russie », Documents pour I'histoire des techniques [En ligne], 14 | $2^{\mathrm{e}}$ semestre 2007, mis en ligne le 09 novembre 2010, consulté le 08 septembre 2020. URL : http://journals.openedition.org/dht/1158 ; DOI : https://doi.org/10.4000/ dht. 1158 


\title{
Le transfert technique et ses avatars : le cas de la Russie
}

\author{
Irina Gouzévitch \\ Centre Alexandre Koyré, EHESS
}

\begin{abstract}
RésumÉ
Ce texte n'est pas un article à proprement parler même s'il en recèle les traits. II s'agit plutôt d'un survol qui tente de présenter, dans ses plus grandes lignes, une thèse consacrée à la problématique très prenante de la circulation du savoir technique. Les divers aspects de cette problématique font aujourd'hui l'objet d'examens attentifs de la part des historiens de plusieurs disciplines. Ils concernent aussi bien des échanges entre les pays que la mise sur pied des réseaux de circulation embrassant des aires culturelles et géographiques multiples, voire des continents tout entiers. Dans le cas que j'étudie, un exemple concret a été retenu, celui de la Russie moscovite et impériale, et de ses relations techniques complexes avec l'Europe, ceci sur une période longue de trois siècles. Ce regard permet de voir le processus en évolution, des débuts encore timides à une véritable percée cognitive, mais aussi d'en suivre les diverses filières, de la circulation des hommes et des objets techniques à celle, parfois plus sinueuse, des idées et des textes et d'en évaluer l'impact.
\end{abstract}

Résumés et mots clés en anglais sont regroupés en fin de volume, accompagnés des mots clés français.

\section{Le transfert technique vers la Russie : problématiques et interrogations}

De la Moscovie d'Ivan III (1465-1505) à l'Empire russe d'Alexandre ler (1801-1825), le transfert massif des connaissances occidentales apparaît comme un facteur important dans la construction de l'État russe. Les deux grandes vagues du transfert, celles qui se produisirent à la charnière des $x v^{e}$ et $x v l^{e}$ siècles, puis à la charnière des $X V I^{e}$ et $X V I I I^{e}$ siècles, coïncidèrent avec la naissance des deux structures étatiques. La première survint au moment où la Principauté de Moscou se transformait en État moscovite (fig. 1). La seconde eut lieu lors de la transformation de celui-ci en Empire russe. Dans les deux cas, le transfert fut initié pour satisfaire à des besoins techniques: réorganiser l'armée, ériger les fortifications, créer la flotte militaire... Dans les deux cas, il aboutit à la réorganisation globale du mode de vie existant. On est donc en droit de s'interroger sur la nature de ces réformes et sur leur alternance. En effet, dans quelle mesure les réformes ont-elles initié le transfert des savoirs et déterminé son échelle ? Et dans quelle mesure ont-elles été elles-mêmes portées et affinées par ce transfert ? En définitive, l'interrogation principale de cette étude se résume ainsi : quel a été le

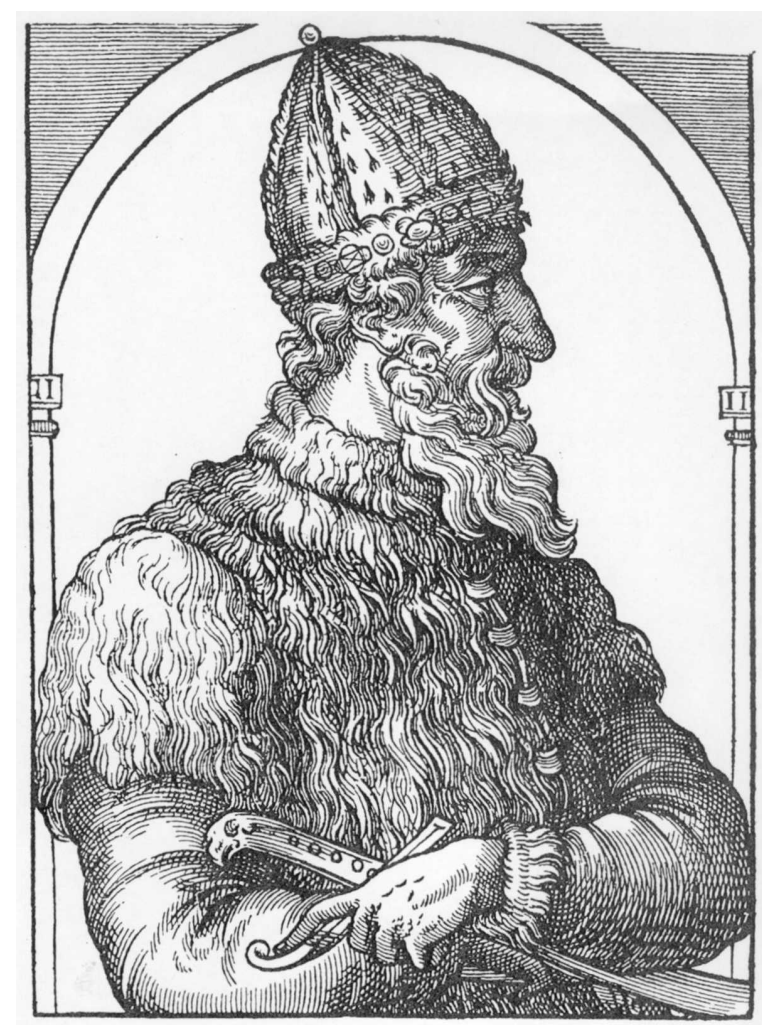

Fig. 1. Ivan III. Gravure du xvI siècle. 
rôle du transfert des savoirs techniques et scientifiques dans la construction de l'État russe ?

Le processus d'acculturation des savoirs techniques occidentaux sur le sol russe est au centre de cette étude. Il se trouve au carrefour de deux problématiques : celle du transfert des savoirs (ses visées, ses filières, ses acteurs, sa nature) et celle des modalités de son accueil sur place (conjonctures, enjeux, mécanismes de sélection, institutions). Bien que l'attention principale soit portée sur les techniques, le savoir véhiculé vers la Russie concernait les domaines les plus divers de l'activité humaine, et pour comprendre le résultat sommaire de ce processus, la dimension culturelle du transfert devait être prise en compte.

La logique de la recherche tentée dans cet esprit m'a conduite à entreprendre une étude pluridisciplinaire qui a débordé le cadre strict de l'histoire des techniques proprement dite. Elle m'a également incitée à considérer ce phénomène sur une période suffisamment longue pour pouvoir en dégager les continuités et les traits spécifiques.

Le pari était difficile et les difficultés étaient grandes. En présentant brièvement les caractéristiques principales de ce travail, à savoir : sa problématique ; sa structure ; ses limites chronologiques et la méthodologie employée - je vais évoquer ce que j'ai ressenti moi-même comme ses difficultés et ses insuffisances.

\section{Les prolégomènes}

La réflexion s'appuie sur un travail de longue haleine mené en collaboration avec Dmitri Gouzévitch, d'abord en URSS, puis en France. En ce qui concerne la Russie, l'étude s'inscrit dans la vague des travaux des années 1990, lorsque les effets de la glasnost gorbatchévienne ont autorisé l'ouverture de nouveaux chantiers de recherche historique. Cela nous a permis de mener à bien I'étude engagée au milieu des années 1980 sur le rôle des experts techniques français ayant exercé en Russie au $x x^{e}$ siècle $^{27}$. Durant notre travail en URSS, la problé-

27. Les différents aspects de cette étude ont fait l'objet de publications. Voir, par exemple : avec Gouzévitch D., Guillerme J., Vérin H., "Gospodin Rokur, kotorogo â lûblû ... » : Antuan Rokur de Šarlevil'. K 200-letiû so dnâ roždeniâ ["Monsieur Raucourt que j'aime ... » : Antoine Raucourt de Charleville. Pour le bicentenaire de la naissance], Voprosy Istorii Estestvoznaniâ i Tehniki, nº 3, 1989, pp. 76-88 ; avec Gouzévitch D., « Françuzskie ingenery - tchleny Peterburgskoj Akademii nauk » [Les ingénieurs français - membres de l'Académie des sciences de Saint-Pétersbourg], matique a été formulée en termes de relations bilatérales ou d'influences étrangères par excellence. Vue de "l'intérieur », toute autre version paraissait alors inabordable ${ }^{28}$. Le projet de recherche rédigé dans cet esprit nous a menés en 1991 en France où, progressivement, il a changé de dimension et d'envergure.

Ainsi, grâce aux recherches documentaires menées pendant dix ans dans les archives et les bibliothèques françaises, espagnoles, italiennes, britanniques, néerlandaises et allemandes, grâce aussi aux multiples contacts avec des collègues des différents pays, l'étude a perdu son caractère uniquement bilatéral pour s'étendre à d'autres aires géographiques. En outre, on a pu établir entre celles-ci et la Russie des liens beaucoup plus complexes et multiples que ceux entrevus jusqu'alors ${ }^{29}$. La nature de ces liens étant indissociable du processus de transfert des connaissances, j'ai été amenée à m'interroger sur ses modalités et, notam-

Deâteli russkoj nauki XIX - pervoj etverti xx vekov : Obzor literatury $i$ istotchnikov, Vyp. I, c. II, Saint-Pétersbourg., SPF IIET AN SSSR, 1991, pp. 74-109 ; avec Gouzévitch D., « Les contacts franco-russes dans le monde de l'enseignement supérieur technique et de l'art de l'ingénieur », Cahiers du monde russe et soviétique, 1993, vol. XXXIV (3), pp. 345-368; « Technical higher education in nineteenth-century Russia and France : some thoughts on a historical choice », History and Technology, 1995, vol. 12, pp. 109-117 ; "L'Empire russe : centre ou périphérie ? », dans Gouzévitch I., Bret P., éd., Naissance d'une communauté internationale d'ingénieurs (1 ${ }^{\text {re }}$ moitié du XIx siècle) : Actes de la journée d'étude 15-16 décembre 1994 (CRHST, CSI), Paris, 1997, pp. 37-44 ; avec Gouzévitch D., "Le phénomène des "ingénieurs-résidents" : reconnaissance légale ou espionnage technique ? », dans Guillerme A., éd., De la diffusion des sciences à l'espionnage industriel : $x v^{e}-x x^{e}$ siècles, Actes du colloque de Lyon (30-31 mai 1996) de la SFHST, Cahier d'histoire et de philosophie des sciences, $\mathrm{n}^{\circ} 47$, Paris, SFHST, 1999, pp. 159-181 ; avec Gouzévitch D., « Les corps d'ingénieurs comme forme d'organisation professionnelle en Russie : genèse, évolution, spécificité : $x \|_{11}^{e}$ et $x x^{\mathrm{e}}$ siècles », Cahiers du monde russe, t. XLI, n 4, 2000, pp. 569-614.

28. Sur cette question, voir les détails dans : Gouzévitch I., "Quelques réflexions à propos du "modèle de l'École polytechnique" », SABIX, n²6, déc. 2000, pp. 38-46.

29. Le résultat le plus consistant de ce séjour en Europe a été la constitution d'une importante banque de données composée des sources primaires russes et occidentales auxquelles s'est jointe une collection d'ouvrages multilingues comportant quelques milliers de titres. La plupart d'entre eux ont été inclus dans l'appareil référentiel de la thèse. Dans l'ensemble, la bibliographie sur laquelle elle s'appuie totalise 2626 titres, présentés en deux volets : alphabétique et thématique, sans compter les sources d'archives qui forment une liste à part. L'objectif de cet article étant de présenter ma recherche dans ses plus grandes lignes, j'ai tenu à ne maintenir ici que les références renvoyant soit aux propos les plus importants soit aux citations. 
ment, à me pencher sur le problème plus général de la constitution des réseaux de sociabilité professionnelle et de leurs modes d'interaction.

En effet, comprendre comment émerge et se met en place un réseau professionnel qui étend son action au-delà des frontières nationales ne signifie-t-il pas, avant tout, se rendre compte de la façon dont éclôt et se forge, à travers la multitude et la variété des pratiques historiques, une identité collective perçue comme telle par l'ensemble des hommes du métier ? L'intégration de tel ou tel pays dans le réseau en question s'opère à une vitesse très variable. Ce processus se déroule suivant des modalités qui, à chaque fois, sont conditionnées par nombre de facteurs essentiels - tels la localisation géographique du pays, son type d'organisation étatique, son niveau de développement économique et culturel, son degré d'étanchéité et de réceptivité, la stratigraphie de la société, le profil social des groupes concernés, enfin, la religion - combinés de façon spécifique. Ces mêmes modalités déterminent la durée et la continuité de chacune des trois phases de l'interaction (toutes conventionnelles que soient les limites entre elles) - le transfert, la circulation et l'échange des connaissances et des pratiques professionnelles -, processus visant spontanément à diffuser I'information sur certains types de savoirs qui, en matière d'architecture, d'art de l'ingénieur et de savoir technique s'est engagé à l'échelle de l'Europe à partir de la Renaissance. Cet effort de globalisation est inséparable d'un autre (qui souvent l'entrave, le freine ou le corrige), celui de l'assimilation et de l'harmonisation de ces nouvelles pratiques et enseignements avec les traditions locales, autrement dit leur acculturation ${ }^{30}$. La Russie, dont I'histoire des techniques et des techniciens semble cumuler d'essentielles problématiques et controverses du processus d'auto-identification professionnelle en prise et en négociation constantes avec I'idéologie au pouvoir, m'a paru suffisamment représentative, par le jeu de facteurs qui la déterminent, pour restituer, à travers son exemple, la genèse de cette interaction transnationale dans tout ce qu'elle peut contenir de typique et d'original.

Ainsi, l'épreuve de la globalisation n'a pas été vaine. Elle m'a suggéré que cette spécificité nationale tant de fois mise en exergue n'avait rien d'exceptionnel en soi et que nombre d'autres pays avaient traversé au cours de leur histoire des épreuves du même ordre. Elle

30. Sur ces sujets, voir: Dolby R.G.A., "The transmission of science », History of science, vol. XV, 1997, pp. 1-43. m'a fourni une grille d'interrogations permettant de structurer cette étude, en la sortant en même temps du cadre d'une simple histoire nationale. Elle m'a permis, enfin, de formuler le sujet, le titre et le cadre chronologique de ma thèse.

\section{Transfert des connaissances : un sujet complexe}

Ayant formulé la problématique de la thèse en termes de transfert des connaissances, je me suis proposé d'examiner ce phénomène dans sa complexité, en étudiant ses mécanismes, ses modalités et ses agents. Le transfert est un terme d'action, et j'ai explicitement privilégié sa dimension spatiale et unidirectionnelle : I'information est véhiculée d'une aire géographique à l'autre. Dans certains contextes, le terme « transfert » peut être remplacé par d'autres, dont le sens exprime une nuance particulière, par exemple, la « traduction », qui privilégie la dimension linguistique ; la « circulation », qui souligne le caractère continu du mouvement ainsi que ses trajectoires complexes ; "I'héritage », qui met en évidence sa dimension temporelle, etc.

Le «transfert» fait également partie de deux couples terminologiques conceptuels autour desquels s'organise la recherche : «transfert et État », « transfert et réseau ». II est donc indispensable d'expliquer comment je les comprends. Le mot «État » est utilisé dans ce travail pour désigner « un groupement humain soumis à une autorité souveraine qui s'exerce sur l'ensemble d'un peuple et d'un territoire ${ }^{31}$ ». Le " réseau » est pris dans son acception large : I'ensemble des éléments communicants entre eux ${ }^{32}$.

Le transfert est un processus et en tant que tel il s'effectue par filières, autrement dit par des voies et des modes divers qui assurent le déplacement. Les filières ont leur hiérarchie selon qu'il s'agit des hommes ou des objets. Les filières du premier type regroupent les migrants quel que soit le motif de leur mobilité : professionnel ou économique, confessionnel ou politique. Leur appartenance professionnelle est elle aussi très variable : hommes de métier et ingénieurs, mais aussi architectes et peintres, écrivains et médecins, voyageurs

31. Dictionnaire historique de la langue française, sous la dir. d'Alain Rey, t. 1, Paris, Dictionnaires Le Robert, 1995, pp. 13211322.

32. Grand Larousse encyclopédique en dix volumes, t. 9, Paris, Librairie Larousse, 1964, p. 187. 


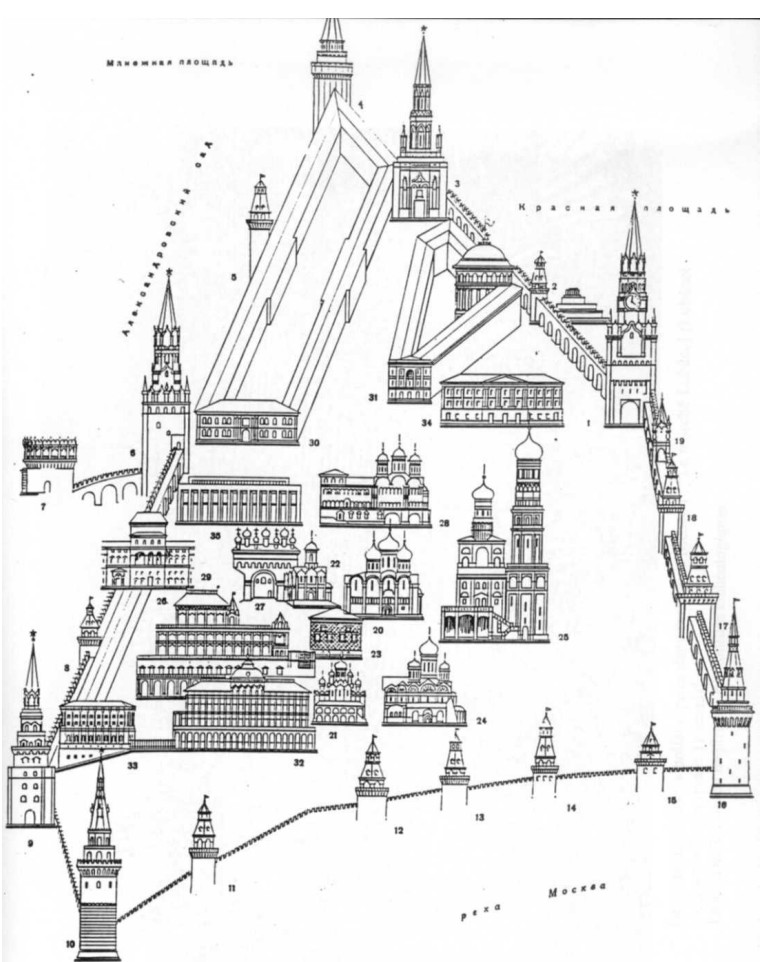

Fig. 2. Travaux des Italiens dans le Kremlin de Moscou. Il'jin M.A. Moskva, Moscou : Isskusstvo, 1963, p. 19.

et hommes d'armes, diplomates et commerçants... Les filières du premier type comprennent également les voyages scolaires et exploratoires, les stages d'étude et de perfectionnement et la reconnaissance (voire I'espionnage) technique. Les filières du second type véhiculent l'information formalisée sous des formes diverses: textes et dessins, outils de travail et instruments de mesure, produits artisanaux et industriels. Au fur et à mesure qu'elles se multiplient et se diversifient, les filières de transfert forment des réseaux moyennant lesquels les connaissances produites dans tel ou tel endroit peuvent circuler dans l'Europe entière. C'est au cours de cette mise en réseau que le transfert dans un sens cède sa place aux échanges des savoirs et à leur circulation. En Russie, ce processus s'acheva vers le début du XIX siècle. Les réseaux de communication professionnelle s'y constituent donc au moment où s'y achève l'institutionnalisation du métier de l'ingénieur.

\section{Les «pourquoi» d'une chronologie longue}

Chronologiquement, l'étude embrasse une période de trois siècles et demi. Elle débute à la fin du $x v^{e}$ siècle et s'arrête au début du XIx siècle. Le choix de ce cadre chronologique a été dicté par la logique même du su-

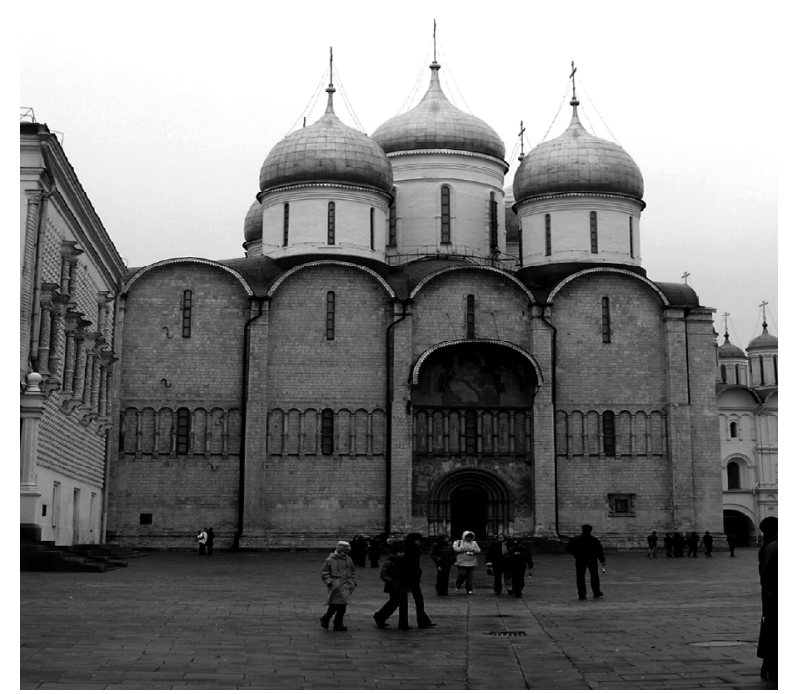

Fig.3. La cathédrale de la Dormition (Kremlin de Moscou). Ph. ( I. Gouzévitch

jet. En effet, la venue des Italiens en Russie (1469-1539) coïncide avec quelques événements dont la conjonction a fait de cette période un moment clé de l'histoire du pays, comme la consolidation des terres russes autour de Moscou, la chute de Constantinople (1453) qui fit de celle-ci l'héritière spirituelle de Byzance et le conflit qui opposa au sein de l'élite dirigeante moscovite les partisans et les contestataires de l'Union florentine avec le pape ${ }^{33}$. Dans ce contexte tendu, inviter les Italiens pour ériger la principale cathédrale du pays - la cathédrale de la Dormtion, dans l'enceinte du Kremlin - apparaît comme un geste profondément symbolique traduisant la volonté du souverain de défier le pouvoir de l'Église tout en affirmant le sien (fig. 2 et 3). Revoir dans cet esprit les aboutissements de l'époque pionnière d'Ivan III m'a semblé une bonne entrée en matière. Les réalisations de l'époque pétrovienne (1689-1727) constituent le noyau central du travail ${ }^{34}$. Un siècle en-

33. L'Union florentine (1438) était une tentative de réunir les branches orientale et occidentale de l'église chrétienne sous l'égide du pape ; ce fut l'ultime tentative désespérée engagée par l'Empire byzantin dans l'espoir d'obtenir l'aide de l'Occident dans sa lutte contre les Turcs.

34. Le règne officiel de Pierre Ier (avec son frère Ivan) débuta en 1682, mais son règne effectif ne commença qu'après la chute de la régente Sofia en 1689. Pour désigner la fin de l'époque pétrovienne, j'ai choisi non pas la date de la disparition physique du réformateur (1725), mais la fin du règne de Catherine Ire, son épouse et héritière au trône qui, de son vivant, poursuivit sa politique. 


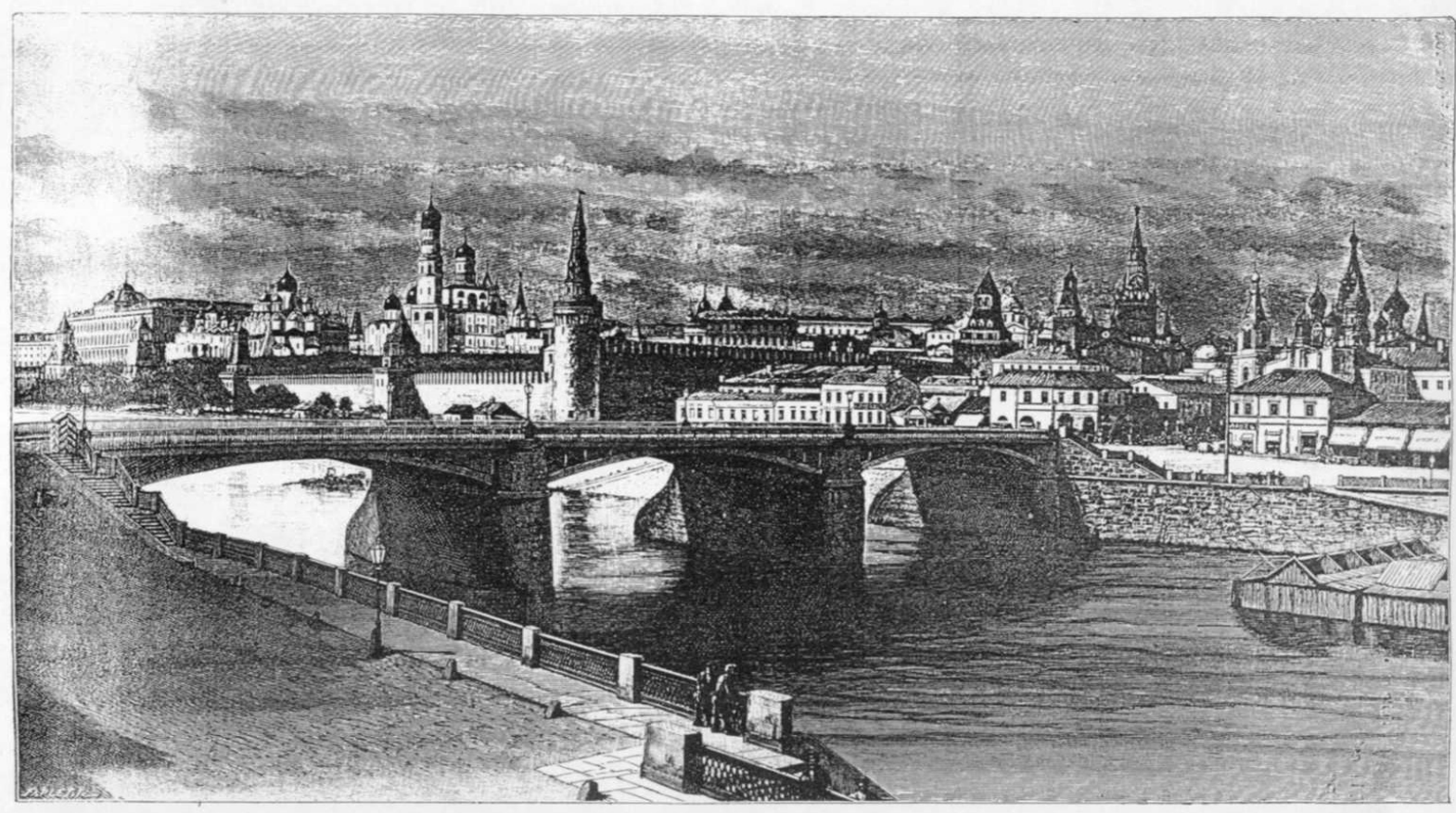

Fig. 4. Le Kremlin de Moscou. Lithographie des années 1880, Journal pittoresque.

tier s'avéra nécessaire pour épuiser le potentiel évolutif accumulé durant cette période révolutionnaire ${ }^{35}$. Afin de mettre en exergue cette continuité qui déborde les limites temporelles fixées par le calendrier, j'ai choisi de désigner cette longue période en termes de « grand $\mathrm{xVIII}^{\mathrm{e}}$ siècle ». Pour la Russie, il commence au milieu des années 1690, avec les campagnes d'Azov, et s'achève au début des années 1810, avec la défaite de l'invasion napoléonienne.

\section{L'étude du transfert en trois volets}

L'étude comprend trois parties dont le découpage obéit au principe chronologique. Cependant, les événements n'évoluent pas à la même vitesse dans la Moscovie ancienne, dans la Russie pétrovienne ou dans l'Empire russe du $x \mathrm{VIII}{ }^{\mathrm{e}}$ siècle. Parfois la pression des événements est telle que le temps historique semble se comprimer.

35. Voir, par exemple, chez Kirillov : «Le potentiel créateur du programme [de l'époque pétrovienne] était si grand que pour accomplir les projets architecturaux et urbanistes de Pierre ler, les efforts de quelques autres générations s'avérèrent nécessaires. L'achèvement de l'expérience amorcée par lui tombe [...] sur la deuxième moitié du xvıll siècle lorsque s'installa en Russie la culture des Lumières. " Kirillov V., " Na perelome ot srednevekov'â k novomu vremeni : Russkaâ model' evropeizacii arhitektury », Vestnik Moskovskogo universiteta, Ser., Istoriâ, 1997, n 2, p. 60.
La structure du travail tient compte de cette accélération. La première partie est consacrée à l'épisode initial du transfert du savoir occidental vers la Moscovie et à ses aboutissements durant l'époque pré-pétrovienne.

Elle débute par le survol de la première percée italienne qui ouvrit le pays aux réalisations techniques de la Renaissance (fig. 4) ${ }^{36}$. Le processus de transfert est ensuite examiné du point de vue des modalités concrètes de l'obtention de l'information (mobilité des experts, modes de formations, circulation des objets, des textes et des images, etc.).

L'intensité sans égale de l'époque pétrovienne m'a incitée à consacrer une partie entière à ce règne. Des débuts difficiles à l'essor de la grande œuvre modernisatrice, l'histoire de Pierre ler est particulièrement éclairante du rôle que joue le pouvoir fort dans la politique d'acculturation des innovations (fig. 5). L'histoire de cette époque a fait objet d'études nombreuses. Vus à la lumière du transfert, ces sujets classiques sont pourtant souvent reconfigurés et réarticulés. Cela concerne, notamment, les premières initiatives techniques de ce règne inspirées par les vicissitudes des deux campagnes d'Azov (1695 et 1696) ; la « révolution culturelle » provoquée par la Grande Ambassade (1697-1698), pre-

36. Celles-ci concernent essentiellement les domaines suivants : I'architecture, la fortification, I'artillerie, la fabrication de la poudre, l'art et les techniques de la construction, la prospection minière. 


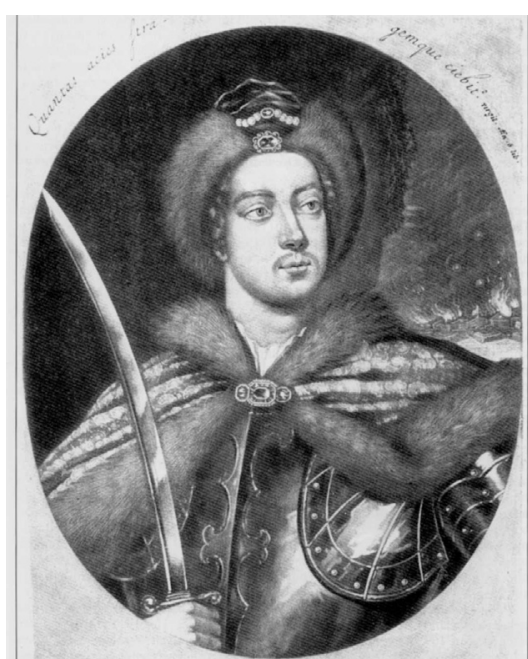

Fig. 5. Pierre ler.

Gravure du premier xvIII siècle.

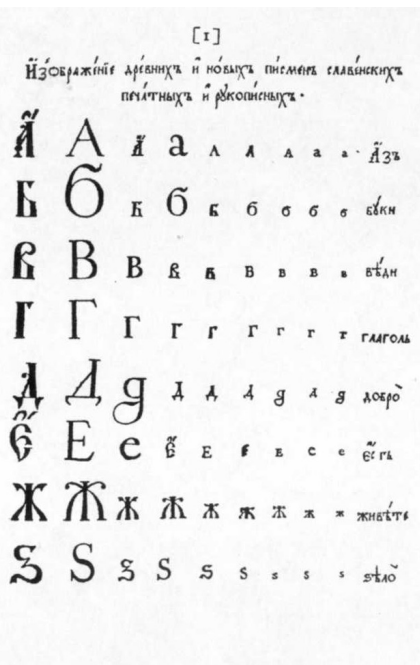

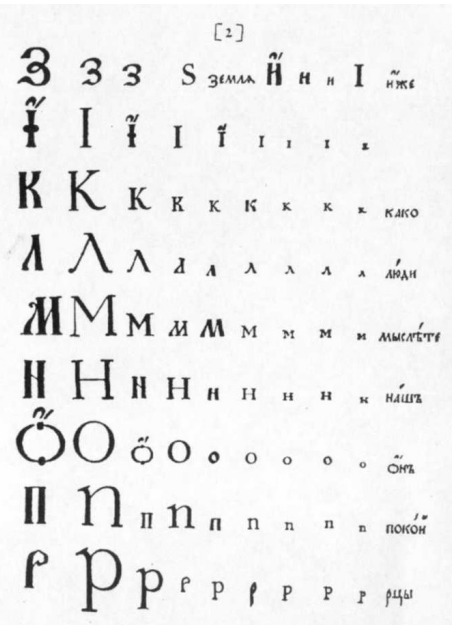

mier voyage du souverain russe en Europe occidentale ; enfin, la mise en place du projet civilisateur avec tout ce que cela implique comme innovation en matière de communication formelle : la promotion du livre laïc et I'introduction de l'alphabet civil, l'adoption de la numérotation arabe et la traduction d'une masse d'ouvrages occidentaux, sans parler de la naissance de la langue russe littéraire et de la mise en route du projet académique (fig. 6).

Dans la troisième partie, les aboutissements de l'époque pétrovienne sont examinés en relation avec I'institutionnalisation du métier d'ingénieur en Russie. L'examen des filières du transfert apporte des éléments aidant à restituer les divers aspects de ce processus : la mise en place de l'enseignement technique et des organismes d'enrôlement professionnel des ingénieurs ; l'intensification de l'activité scientifique et l'effervescence des sciences de l'ingénieur ; le rôle que joua dans ce processus la migration professionnelle. Enfin, le dernier chapitre invite à réfléchir sur les paradoxes de I'histoire qui, tout en ignorant le conditionnel, est malgré tout parfois harcelée par la question des occasions manquées, tel le refus de Catherine II d'accorder le grade de major du service russe au jeune artilleur corse Napoléon Buonaparte ${ }^{37}$.

Les exemples présentés dans l'épilogue permettent de tracer les pistes à suivre dans l'avenir. Le premier $X I x^{e}$ siècle marque en effet un grand jalon dans I'histoire du transfert technico-scientifique relancé par les

37. Miller K., Francuzskaâ emigraciâ i Rossiâ v carstvovanie Ekateriny II [L'émigration française et la Russie sous le règne de Catherine II], Paris, La source, 1931, p. 362. réformes modernisatrices d'Alexandre Ier. La naissance du réseau des grandes écoles et des grands corps techniques, l'émergence des nouvelles disciplines scolaires et des groupes d'ingénieurs-chercheurs compétitifs à l'échelle européenne sont autant d'aboutissements de cette politique qui permettent d'affirmer que l'ingénieur moderne est né en Russie à cette époque-là38

\section{L'histoire des techniques russe à l'épreuve de la problématique du " transfert »}

Aborder le sujet du point de vue des rapports entre le transfert technique et la politique signifiait pour moi,

38. Ces sujets ont été développés par l'auteur dans une série de publications : avec Gouzévitch D., « Transfert de technologies en matière de travaux publics en Russie : quête aux innovations et choix politiques, 1800-1850 », dans Tissot L., Veyrassat B. éd., Technological trajectories, markets, Institutions : industrialized countries, 19th-20th centuries: from context dependency to part dependency [Trajectoires technologiques, marchés, institutions: les pays industrialisés, $19^{e}-20^{e}$ siècles : de la dépendance du contexte à la dépendance de sentier], Bern, Peter Lang, 2001, p. 91-101; «Augustín de Betancourt : el modelo de la comunicación profesional de los engenieros a finales del siglo xVIII y principios del XIX », trad. Carlos M. Collantes, dans Montesinos J., Ordoñez J., Toledo S. éd., Ciencia y Romanticismo [Maspalomas, Septiembre 2002], La Orotava, Fundación Canaria Orotava de Historia de la Ciencia, 2003, p. 303-327 ; avec Gouzévitch D., «Travelling Interchanges Between the Russian Empire and Western Europe : the Travels of Engineers During the First Half of the Nineteenth-Century ", dans Simões A., Carneiro A., Diogo M.P. éd., Travels of learning : a geography of science in Europe, Boston, Kluwer acad. publ., 2003, p. 213-231. 


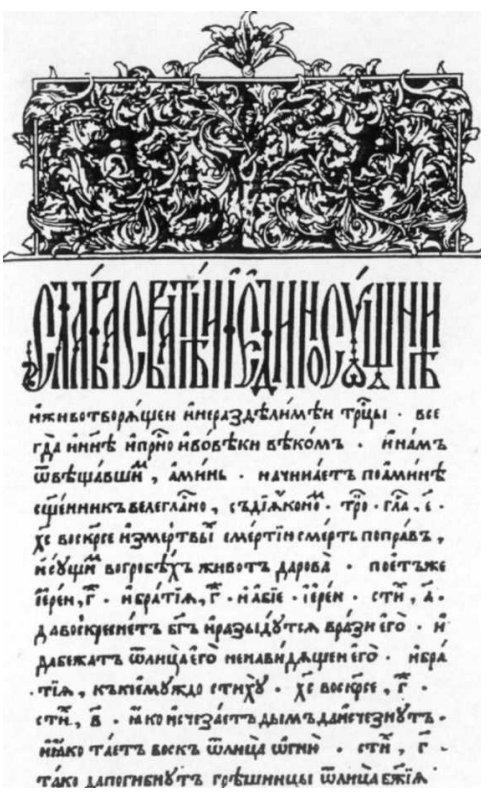

\section{ГEOMETPIA C $A$ A B E H C K I \\ SEM $\lambda E M$ DP IE}

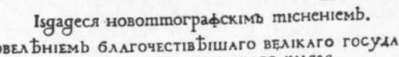

Петра Aлексіевіча,

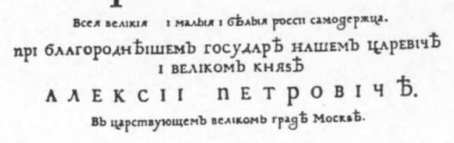

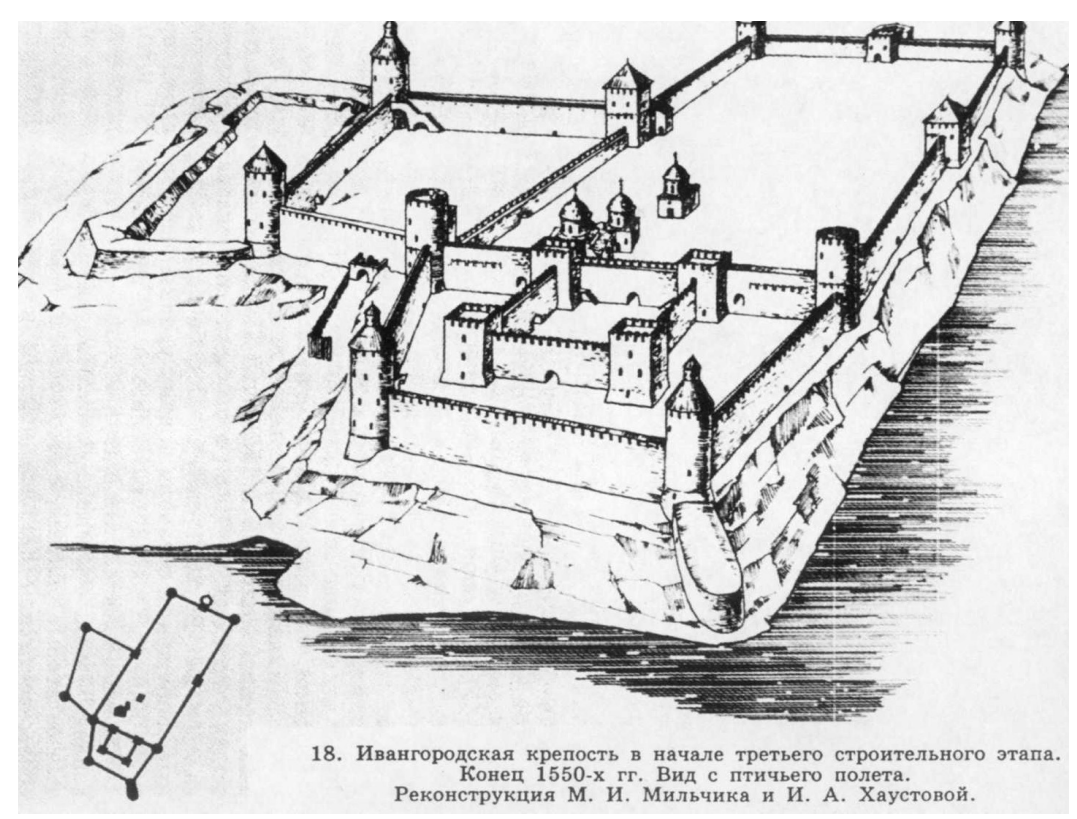

Fig. 7 (à gauche). Naissance du livre russe laïc. Pages de titre d'ouvrages édités en 1591 et 1708.

Fig. 8 (ci-dessus). La forteresse d'Ivangorod, reconstruction par M. Mil'chik et I. Hrutova. Novgorodskij istoricheskij sbornik, n5/15, 1995 p. 196.

d'une part comprendre comment les connaissances sur les techniques occidentales se diffusaient et s'acculturaient dans les terres russes, d'autre part réarticuler le chantier de travail par rapport à un certain nombre de représentations historiographiques classiques concernant les rapports de la Russie avec l'Occident.

Beaucoup d'historiens dont j'ai cité les propos au cours de cette recherche ont, notamment, insisté sur le caractère " autre » et " différent » de la culture russe pour expliquer la perception des innovations techniques transférées. L'argument en soi n'a rien de surprenant dans la mesure où toute culture nationale revendique son identité. On pourrait donc l'admettre si l'explication n'était pas souvent reléguée dans la sphère de l'irrationnel et de l'inconnaissable. Selon l'analyse tentée dans l'étude présentée, si cette fameuse "différence » existe effectivement, loin de ressortir d'une " âme » russe présupposée, elle a des causes tout à fait repérables et matérielles. Certains exemples détaillés dans le corps du texte l'ont clairement démontré, telle l'histoire de l'introduction de l'art de l'imprimerie.

Pendant plus d'un siècle, depuis l'invention de Gutenberg, la Moscovie opposa à cette innovation une résistance obstinée. Vers la fin du $x v l^{e}$ siècle, cet art prit enfin racine sous l'égide exclusive de l'Église orthodoxe. Le caractère sacré du texte, l'autorité universelle du livre qui ignore toute expression personnelle sont autant d'arguments qui expliquent l'usage restreint de la production polygraphique réduite en Russie au seul domaine du spirituel ${ }^{39}$. II manquait par ailleurs quatre facteurs principaux qui auraient pu favoriser son accueil : un milieu réceptif, un commanditaire, un public et un mobile. II existait en même temps deux facteurs qui contribuèrent à ce qu'on s'y opposât : la méfiance face aux innovations d'origine latine et la censure idéologique centralisée des moyens d'information. Le milieu réceptif susceptible de comprendre l'importance médiatique du livre - celui des judaïsants - fut anéanti. Les lecteurs potentiels, peu nombreux dans ce pays où la culture scolaire était encore embryonnaire, se contentaient du livre manuscrit, paradoxalement beaucoup moins cher que le livre imprimé. L'initiative des particuliers n'était pas suffisante pour lancer une industrie dont l'État seul se voyait décideur. Or, pour que l'État y investît, un mobile puissant était indispensable. Et le livre russe laïc - technique, militaire et codificateur

39. Voir à ce propos: Pan enko A. M., Russkaâ kul'tura v kanun petrovskih reform [La culture russe à la veille des réformes pétroviennes], Leningrad, Nauka, 1984, 206 p. 
- naquit sous Pierre ler des impératifs de la guerre du Nord (1700-1721) (fig. 7).

Les exemples de la fortification et de l'artillerie permettent d'illustrer un autre trait de la culture traditionnelle : sa capacité à s'approprier une innovation transférée en faisant d'elle, à son tour, une tradition immuable (fig. 8). En effet, un siècle après la percée italienne, on reproduisait encore dans ces domaines des techniques obsolètes alors que des inventions locales intéressantes n'existaient que sous forme d'ouvrages ou de pièces uniques. Parmi les causes de cette controverse, trois me semblent prépondérantes. Premièrement, le caractère intermittent des grandes percées informationnelles. Deuxièmement, l'absence d'une infrastructure de production industrielle quelque peu développée. Troisièmement, la carence des moyens de communication formelle et l'anonymat de l'inventeur. C'est à cette mutité du maître anonyme et isolé que Pierre ler opposa le « creuset » des chantiers navals de Voronezh et de la Baltique où les maîtres charpentiers de l'Europe entière travaillaient côte à côte (fig. 9). L'information technique relative à la construction des vaisseaux cessa ainsi d'être un secret de maître. Cependant, pour que I'ancienne tradition soit rompue définitivement, il fallut attendre que se mettent en place des institutions et des systèmes de communication nouveaux : les établissements d'enseignement spécialisé, les corps d'ingénieurs et le livre technique. C'est de la convergence de ces facteurs que naquit un groupe de spécialistes techniques dont I'identité collective fut porteuse de valeurs différentes, qui privilégiaient non pas une performance tacite et inédite, mais une activité productive d'utilité publique ouverte à une large diffusion. L'émergence de cette nouvelle communauté d'ingénieurs constitue, à mon sens, l'un des aboutissements majeurs des réformes pétroviennes et l'un des piliers principaux de la puissance technique sur laquelle s'appuya le nouvel ordre impérial.

La comparaison des deux époques historiques, celles d'Ivan III et de Pierre ler, permet de réfléchir sur un autre paradoxe du transfert : le faible rendement de I'assimilation locale par rapport à la masse de l'information transférée. Ainsi que les exemples de la fortification et de l'artillerie le démontrent, quand bien même ce transfert intervient dans la mise sur pied des grands systèmes techniques, cela ne garantit guère son afflux ultérieur régulier dans ces domaines. L'époque d'Ivan III offre un bel exemple de l'incohérence entre le transfert massif et I'assimilation partielle et restrictive. Les mécanismes de protection actionnés au sein de la culture

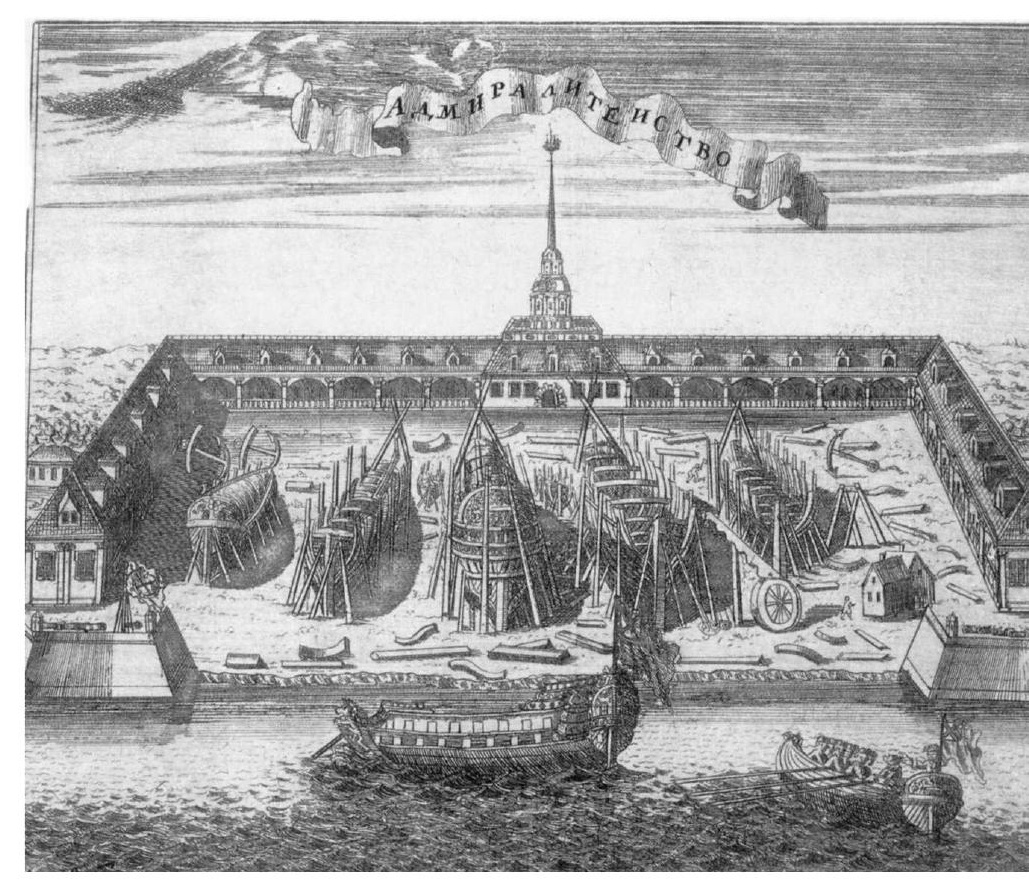

Fig. 9. L'Amirauté de Saint-Pétersbourg . Gravure d’A. Zubov (1717).

nationale ont coupé court à toute tentative d'étendre le contact à la sphère des idées humanistes de la Renaissance. La bataille perdue contre l'Église par le pouvoir séculier décida ainsi des modalités particulières du transfert pour les deux siècles à venir. Seul le savoir concret relatif à tel ou tel domaine des techniques et du militaire avait la chance d'aboutir sans trop d'encombres. Une fois le problème concret résolu, I'intervention s'arrêtait là, et le plus souvent les contacts n'avaient pas de suite à court et moyen termes. Certaines autres innovations connurent en Russie un sort différent : trop performantes et trop coûteuses, elles n'existaient que sous forme de jouets exotiques dont l'extension se faisait longtemps attendre. Pour que ces innovations pussent être acculturées, la circulation continue des connaissances était indispensable. Or, cette circulation ne pouvait être assurée que dans un contexte adéquat, celui-là même qui avait conditionné leur floraison en Europe. Pierre ler finit par réaliser cette tâche lorsque, parti en Occident en quête de solutions techniques relatives à la construction de la flotte, il en ramena une " civilisation ${ }^{40}$ ». Cette démarche assura à ses initiatives

40. Pour la démonstration détaillée, voir, outre le chapitre respectif de la thèse : Gouzévitch D., « Le Premier voyage de Pierre Ier en Europe (1697-1698) et son rôle dans le transfert des connaissances techniques et scientifiques vers la Russie », DEA en histoire et civilisation, EHESS, sous la dir. de W. Bérélowitch, soutenu en septembre 2000 ; Gouzévitch D., Gouzévitch I., Velikoe posol'stvo, 
techniques une implantation solide et une évolution durable ; elle permit d'aller bien au-delà des solutions techniques ponctuelles; elle rompit la coutume d'une action de transfert isolée ; elle contribua à modifier la nature même des rapports de la Russie avec I'Occident ; elle conféra à la culture impériale naissante son caractère européanisé.

Parmi les questions soulevées par ce travail, I'une semble cruciale : comment la culture russe arriva-t-elle à faire sien ce « contexte» ? pourquoi n'a-t-elle pas extirpé de son sein les éléments étrangers imposés de force et profondément contraires à sa nature (par exemple la nouvelle capitale ou l'Académie des sciences) ? Cette interrogation est révélatrice d'une controverse que seule l'analyse attentive de l'usage de l'expression « la culture russe » peut aider à élucider. La comparaison des résultats du transfert aux époques d'Ivan III et de Pierre ler met sur la piste d'une réponse : ne s'agirait-il pas d'une confusion provoquée par la notion polysémique du terme ? II semble, en effet, que les contestataires de certaines initiatives pétroviennes omettent de faire la différence entre deux nuances du mot « russe » qui désignent en réalité deux phénomènes différents : russkij, c'est-à-dire « russe » dans le sens ethnique du mot, et rossijskij, c'est-à-dire « russe » dans le sens politique, impérial du mot. À l'époque d'Ivan III, il s'agissait de la culture du premier type, et cette culture recula devant l'afflux des connaissances occidentales profondément étrangères à sa nature. Cependant, durant le siècle suivant, il se passa bien des choses. Les conquêtes territoriales réalisées entre 1552 et 1664 (les khanats de Kazan et d'Astrakan) firent de la Moscovie un empire de facto. La culture russe monoethnique commença à muter en conséquence en acquérant les traits d'une (mega)culture impériale assise sur une multitude de cultures ethniques diverses. Le savoir occidental transféré vers la Russie durant l'époque pétrovienne fut donc assimilé par une culture assouplie et plus réceptive car disposant déjà des mécanismes d'assimilation et d'une expérience de contacts plus poussée ${ }^{41}$.

Sans épuiser toutes les problématiques soulevées par la thèse, ces quelques remarques permettent néan-

Korni Peterburga [La Grande Ambassade, dans la série Les Racines de Pétersbourg], Saint-Pétersbourg, Feniks, 2003, 311 p.

41. Cette hypothèse testée dans la thèse a été formulée dans : Gouzévitch D., Kentavr ili k voprosu o binarnosti russkoj kul'tury [Centaure ou sur la binarité de la culture russe], AIRO - Nautchnye doklady i diskussii : Temy dlâ XXI veka-Vyp. 7, Moscou., AIRO-XX, 2000, $80 \mathrm{p}$. moins d'illustrer le potentiel heuristique d'une étude qui, à travers une histoire du transfert des savoirs techniques examiné dans un contexte socioculturel large, est susceptible d'apporter un regard différent sur I'histoire et la civilisation d'un pays.

\section{Quelques mots à propos des difficultés}

Les difficultés rencontrées au cours de ce travail se situent à plusieurs niveaux.

La première, à caractère estimatif, découle de la nature même de l'étude. Dans la mesure où celle-ci porte sur le transfert des connaissances occidentales vers la Russie, mon attention a été centrée sur les domaines où de telles connaissances étaient sollicitées puisqu'elles manquaient dans le pays d'accueil. En suivant la piste des apports européens, j'ai été amenée à évoquer le contexte dans lequel on avait fait appel à eux et à constater leur caractère pionnier et stimulant. Malgré l'effort de neutralité, cette démarche risquait, par moments, de donner l'impression d'une vision catastrophiste de quelqu'un obsédé par le complexe de la pauvreté russe et de la performance européenne. L'épreuve de l'impartialité est, en effet, extrêmement difficile à assumer. Consciente de ce fait, je tiens à formuler un avis qui ressort de l'étude réalisée. D’une part, elle m'a appris à relativiser la notion même de performance pour laquelle, de toute façon, il n'y a pas de critère absolu. Ce qu'on identifie comme «le meilleur » rétrospectivement ne l'était pas nécessairement aux yeux des acteurs des événements. Fautil les accuser de cécité lorsque certains de leurs choix nous paraissent aujourd'hui aléatoires ? Ne vaut-il pas mieux reconnaître qu'ils agissaient selon le bon sens qui était le leur en empruntant ce qui répondait aux besoins de leur temps voire était conforme aux goûts des décideurs ? La sélectivité n'était-elle pas elle aussi un principe à apprendre ? Et l'étude a démontré que la Russie a mis deux siècles avant d'appliquer ce principe sciemment. La réponse est à rechercher dans la perception historiquement établie de l'Europe par les Russes. La position géographique excentrée de la Moscovie, située au carrefour de l'Europe et de l'Asie, y joua un rôle important. L'héritage byzantin et les valeurs de la spiritualité orthodoxe, qui imprimaient à sa culture un caractère contemplatif et techniquement peu dynamique, avaient également leur part d'influence. Vue de la Moscovie, l'Europe occidentale offrait l'image d'un monde lointain, diffus, redoutable et beaucoup plus 
uniforme qu'il ne l'était en réalité. La politique restrictive vis-à-vis des étrangers qu'on tolère mais dont on se méfie est un revers fréquent de l'histoire du transfert. Dès le $x v l^{e}$ siècle, elle aboutit à la création des zones de résidence destinées à isoler les étrangers hétérodoxes de la population autochtone. C'est au sein d'un tel ghetto, le Faubourg allemand, ou Kukuj, que naquit au $\mathrm{xVI} \|^{\mathrm{e}}$ siècle un certain « mythe de l'Europe » dynamique et technicienne. Cette vision somme toute idéalisée nourrit l'imaginaire du jeune Pierre ler et l'incita, pour la première fois dans I'histoire de la royauté russe, à entreprendre une tournée en Occident. Le transfert massif des connaissances européennes et les réformes fondamentales que suscita ce voyage (la Grande Ambassade) sont à l'origine du caractère européen que prit la nouvelle culture impériale.

Le problème des sources est une autre grande difficulté de ce travail. Résumés de la façon la plus succincte, ces problèmes sont les suivants.

Outre les documents d'archives et les chroniques (très lacunaires pour le $x v^{e}$ et le $x v l^{e}$ siècle), cette étude s'appuie sur les textes à caractère épistolaire et mémorialiste russes et étrangers. Puisque l'un des objectifs de ce travail consistait à réarticuler du point de vue du transfert certains grands événements de I'histoire russe, la question de l'emploi des sources secondaires était également abordée. Étant donné que cette étude consacrée à la Russie a été faite en France, j'ai tenu à y rassembler le maximum des sources russophones relatives aux sujets traités dans le double souci de rendre l'étude plus élaborée et d'informer les historiens de l'existence de cette riche littérature peu connue en Occident.

La dernière difficulté était (et reste toujours) d'ordre terminologique. Elle relève d'une part, de la polysémie des termes utilisés et d'autre part, du problème de leur traduction adéquate. Ce problème est toutefois commun à l'ensemble des historiens.

Que peut nous apprendre I'histoire du transfert des connaissances occidentales vers la Russie?

Le premier constat qui en ressort concerne le caractère récurrent du processus de transfert qui ne tarit pas durant toute la période examinée. L'alternance des périodes plus ou moins intenses est un autre trait typique de ce processus. L'intensité du transfert est tributaire de nombreux facteurs. Elle dépend des conditions de l'État russe à chaque moment donné, notamment de son de- gré d'ouverture et de tolérance religieuse, de l'évolution de ses structures administratives et éducatives, de l'état de son économie, de ses rapports avec les voisins, voire des goûts personnels du monarque. Le transfert des connaissances intervient activement dans la construction de cet État, et ceci à tous les niveaux, de sa symbolique à la combativité de ses troupes, de l'organisation de ses administrations à l'étiquette de la cour.

Les motifs qui, depuis Ivan III, ont incité les gouvernements russes à recourir au transfert des connaissances techniques occidentales, sont d'origines externe et interne. Les premiers sont d'ordre militaire et économique. Les impératifs de la défense allaient souvent de pair avec les intérêts commerciaux : la plupart des guerres que la Russie mena contre l'Occident visaient la liberté du commerce extérieur. Parmi les motifs internes, celui de la consolidation du pouvoir séculier paraît essentiel. Au fil des siècles, le transfert technique fut utilisé pour affirmer son prestige et ses prérogatives vis-à-vis de sa rivale principale, l'Église orthodoxe. Dans cette lutte d'influences, l'Église joua la carte de la protection des valeurs traditionnelles en mettant en route les mécanismes du rejet des innovations européennes. Pour parer cet obstacle, le pouvoir séculier élabora un grand nombre de mécanismes de transfert et d'acculturation des innovations. À l'époque de Pierre ler, ce bras de fer s'acheva par l'échec du clergé. Cependant, I'orthodoxie ne rendit jamais toutes les armes. L'auto-isolement périodique du pays dont ce travail fait également constat ne fut-elle pas redevable de la perpétuation de cette opposition au sein de la société ?

Appliqué au fil des siècles, le transfert des connaissances agit en profondeur. II intervint activement dans le processus de modernisation qui imprima à la nouvelle culture impériale son caractère européanisé. II contribua à rompre l'isolement séculier de la Russie. II stimula ses rapports avec le monde occidental et modifia leur nature. L'intégration progressive de la Russie dans l'espace européen au sein duquel le savoir circule librement est, à mon sens, I'un des aboutissements majeurs de cette politique. Certaines initiatives du XVIII siècle russe vont même au-delà des sources d'inspiration connues. Elles apparaissent plutôt comme la quintessence de l'esprit européen, comme l'incarnation de certains idéaux que I'Europe elle-même ne put alors réaliser pleinement. « Le mythe européen » ne triompha-t-il pas dans cette nouvelle percée? 